\title{
新的锂矿物一一扎布耶石的晶体结构
}

\author{
林月美 张汉卿 郑绵平 \\ (中国地质科学院矿床地质研究所, 北京)
}

关镜词扎布耶石

\section{一、矿物 学}

扎布耶石 (Zabuyelite) 楸是在我国西藏扎布耶盐湖中首次发现的天然碳酸锂新矿物.由 产地而得名. 矿物属单斜晶系, 晶形完好, 呈细长柱状, 晶体无色透明. 比重 $D_{\text {新 }}=2.09 \pm$ 0.01. $\{100\}$ 解理完全. 矿物的化学成分经扣除杂质后, $\mathrm{CO}_{2}$ 为 $48 \%, \mathrm{Li}_{2} \mathrm{O}$ 为 $34 \%$, 其分子 数之比近似 $1: 1$, 因此, 矿物的化学式为 $\mathrm{Li}_{2} \mathrm{CO}_{3}$, 化学成分列于表 1. 扎布耶石与人造碳酸 锂 ${ }^{[2,3]}$ 的 X 射线粉晶衍射线相一致 (见表 2 ).

表 1 扎布耶石化学成分

\begin{tabular}{|c|c|c|c|c|c|c|c|c|c|c|c|c|c|c|c|}
\hline 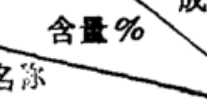 & $\mathrm{Li}_{2} \mathrm{O}$ & $\mathrm{Na}_{2} \mathrm{O}$ & $\mathrm{K}_{2} \mathrm{O}$ & $\mathrm{MgO}$ & $\mathrm{CaO}$ & $\mathrm{Fe}_{2} \mathrm{O}_{3}$ & $\mathrm{M}$ nO & $\mathrm{CO}_{2} \mid$ & $\mathrm{H}_{2} \mathrm{O}^{+}$ & $\mathrm{Cl}$ & so, & $\mathrm{NO}_{3}$ & $\mathrm{~B}_{2} \mathrm{O}_{3}$ & 不溶物 & 总计 \\
\hline 扎布耶石 & 34.20 & 2.49 & 0.75 & 1.81 & 1.24 & 0.88 & 0.017 & 52.70 & 1.88 & 1.09 & 0.57 & 0.44 & 0.10 & 1.89 & 100.057 \\
\hline 碳酸锂 (人造) & 38.82 & 1 & 0.77 & 1 & 1 & 0.057 & 1 & 57.18 & 1 & 0.02 & 0.30 & 1 & 1 & 7 & 97.08 \\
\hline
\end{tabular}

\section{二、晶体结构分析}

用于 $\mathrm{X}$ 射线衍射强度收集和晶胞参数测定的晶体是从数颗优质晶体中, 选取大小为 $0.1 \times$ $0.05 \times 0.03 \mathrm{~mm}$ 的结晶完好、透明度高的单晶体在 $P_{3} / F$ 型四圆衍射仪上进行的. 首先用最 小向量法求得一个合理的单斜晶胞, 晶胞参数经过最小二乘法修正. 最终晶体学参数为 $a=$ 8.361(3) $\AA, b=4.976(2) \AA, c=6.193(3) \AA, \beta=114.69^{\circ}(3), v=234.1(1) \AA^{3}$. 空间群为 $C_{2 h}^{6}=C 2 / C, Z=4 . D_{c}=2.10 \mathrm{~g} / \mathrm{cm}^{3}$. 收集衍射强度数据时, $\mathrm{X}$ 射线经用石墨单色化的 $\mathrm{MoK}$ 辐射. 采用 $\theta-2 \theta$ 扫描方式, 每隔 60 个衍射点重复测定二个参考衍射, 在 $0^{\circ}-80^{\circ}(2 \theta)$ 范围 内测得 1075 个衍射点. 具有独立衍射点为 821 个, 其中可观察点 $F>2.5 \sigma(F)$ 为 534 个点 被用于结构分析. 原始强度数据分别按参考衍射的强度变化进行标度. 衍射强度经过 $\mathrm{Lp}$ 因 子及 $\mu\left(M \circ K_{\alpha}\right)=2.1 \mathrm{~cm}^{-1}$ 吸收校正后, 用直接法 (SHELXTL) 程序求得所有独立的 $\mathrm{Li}, \mathrm{C}$, $\mathrm{O}$ 原子坐标. 然后, 用全矩阵最小二乘法对所有原子坐标及温度因子进行精化修正. 最后获 得偏离因子 $R=0.081$, 加权后 $R_{\omega}=0.076$ (加权方式: $\omega=\left[\sigma(F)^{2}+0.002 F^{2}\right]^{-1}$ ). 原子

本文 1988 年11月10日收到. 
表 2 扎布耶石和碳酸锂 ${ }^{[4]}$ 粉晶分析

\begin{tabular}{|c|c|c|c|c|c|c|c|}
\hline \multicolumn{2}{|c|}{ 扎右耶石 } & \multicolumn{2}{|c|}{ 碳酸锂(人造) } & \multicolumn{2}{|c|}{ 扎布 耶石 } & \multicolumn{2}{|c|}{ 碳能锂（人逍） } \\
\hline$d$ & $r / I_{0}$ & $d$ & $I / I_{0}$ & $d$ & $I / I_{0}$ & $d$ & $I / I_{0}$ \\
\hline 4.15 & 8 & 4.16 & 85 & 1.5144 & 1 & 1.5154 & 6 \\
\hline 3.80 & 1 & 3.80 & 20 & & & 1.5092 & 6 \\
\hline 3.03 & 1 & 3.03 & 25 & & & 1.4980 & 2 \\
\hline 2.92 & 8 & 2.918 & 80 & 1.475 & 2 & 1.4669 & 4 \\
\hline 2.811 & 10 & 2.812 & 100 & & & 1.4622 & 4 \\
\hline 2.62 & 3 & 2.627 & 30 & 1.426 & 2 & 1.4250 & 6 \\
\hline 2.481 & 4B & & & & & 1.4066 & $<2$ \\
\hline 2.42 & 4 & 2.431 & 40 & 1.397 & 3 & 1.3923 & 2 \\
\hline 2.28 & 2 & 2.276 & 20 & & & 1.3879 & 23 \\
\hline 2.256 & 2 & & & & & 1.3754 & 2 \\
\hline 2.11 & 3 & 2.116 & 4 & 1.359 & 1 & 1.3529 & 2 \\
\hline 2.08 & 1 & 2.081 & 8 & 1.330 & 1 & 1.3361 & 2 \\
\hline 1.986 & 1 & 1.910 & 2 & 1.310 & 1 & 1.3132 & 2 \\
\hline & & 1.893 & 2 & & & 1.3042 & $<2$ \\
\hline 1.860 & $2 B$ & 1.867 & 18 & & & 1.2872 & 2 \\
\hline 1.820 & 1 & 1.8205 & 2 & & & 1.2842 & 4 \\
\hline 1.810 & 2 & 1.8121 & 4 & & & & \\
\hline 1.605 & 1 & 1.6208 & 4 & 1.280 & 2 & 1.2807 & 4 \\
\hline & & 1.5958 & 8 & & & 1.2655 & 4 \\
\hline & & 1.5858 & 2 & 1.252 & 2 & 1.2586 & 2 \\
\hline & & 1.5804 & 4 & & & & \\
\hline & & 1.5723 & 4 & & & & \\
\hline 1.565 & 2 & 1.5652 & 8 & & & & \\
\hline & & 1.5469 & 10 & & & & \\
\hline
\end{tabular}

表 3 原子坐标及各向异性温度因子

\begin{tabular}{|c|c|c|c|c|c|c|c|c|c|c|}
\hline 原 子 & $x$ & $Y$ & $z$ & $\mathrm{U}_{\mathbf{1 1}}$ & $U_{22}$ & $U_{33}$ & $U_{23}$ & $U_{13}$ & $U_{12}$ & $\mathbf{U}$ \\
\hline $\mathbf{L i}$ & $-0.3042(5)$ & $0.533(7)$ & $0.3335(6)$ & $\begin{array}{l}0.0130 \\
0.0014\end{array}$ & $\begin{array}{l}0.0140 \\
0.0015\end{array}$ & $\begin{array}{l}0.0242 \\
0.0015\end{array}$ & $\begin{array}{r}-0.0017 \\
0.0012\end{array}$ & $\begin{array}{l}0.0059 \\
0.0012\end{array}$ & $\begin{array}{r}-0.0014 \\
0.0011\end{array}$ & $\begin{array}{l}0.0177 \\
0.0011\end{array}$ \\
\hline C & 0 & $0.671(4)$ & 0.2500 & $\begin{array}{l}0.0079 \\
0.0007\end{array}$ & $\begin{array}{l}0.0100 \\
0.0008\end{array}$ & $\begin{array}{l}0.0126 \\
0.0008\end{array}$ & $\begin{array}{l}0 \\
0\end{array}$ & $\begin{array}{l}0.0028 \\
0.0006\end{array}$ & $\begin{array}{l}0 \\
0\end{array}$ & $\begin{array}{l}0.0107 \\
0.0005\end{array}$ \\
\hline$O(1)$ & $0.1464(2)$ & $-0.637(3)$ & $0.3128(2)$ & $\begin{array}{l}0.0090 \\
0.0005\end{array}$ & $\begin{array}{l}0.0123 \\
0.0005\end{array}$ & $\begin{array}{l}0.0201 \\
0.0006\end{array}$ & $\begin{array}{l}0.0009 \\
0.0004\end{array}$ & $\begin{array}{l}0.0049 \\
0.0004\end{array}$ & $\begin{array}{l}0.0028 \\
0.0004\end{array}$ & $\begin{array}{l}0.0142 \\
0.0004\end{array}$ \\
\hline$O(2)$ & 0 & $0.3222(4)$ & 0.2500 & $\begin{array}{l}0.0114 \\
0.0007\end{array}$ & $\begin{array}{l}0.0084 \\
0.0007\end{array}$ & $\begin{array}{l}0.0351 \\
0.0011\end{array}$ & $\begin{array}{l}0 \\
0\end{array}$ & $\begin{array}{l}0.0067 \\
0.0007\end{array}$ & $\begin{array}{l}0 \\
0\end{array}$ & $\begin{array}{l}0.0193 \\
0.0006\end{array}$ \\
\hline
\end{tabular}

坐标及各向异性温度因子列于表 3. 键长、键角列于表 4、表 5 . 


\begin{tabular}{ll|ll}
\hline $\mathrm{C}-\mathrm{O}(1)$ & $1.294(2)$ & $\mathrm{Li}-\mathrm{O}(1 \mathrm{~b})$ & $2.032(4)$ \\
$\mathrm{C}-\mathrm{O}(2)$ & $1.269(3)$ & $\mathrm{Li}-\mathrm{O}(\mathrm{cc})$ & $1.975(5)$ \\
$\mathrm{C}-\mathrm{O}(1 \mathrm{c})$ & $1.294(2)$ & $\mathrm{Li}-\mathrm{O}(2 \mathrm{a})$ & $1.888(4)$ \\
$\mathrm{O}(1)-\mathrm{Li}(\mathrm{a})$ & $1.943(4)$ & $\mathrm{Li}-\mathrm{Li}(\mathrm{d})$ & $2.710(7)$ \\
$\mathrm{O}(1)-\mathrm{Li}(\mathrm{c})$ & $2.032(4)$ & $\mathrm{Li}-\mathrm{Li}(\mathrm{f})$ & $2.994(8)$ \\
$\mathrm{O}(1)-\mathrm{Li}(\mathrm{e})$ & $1.975(5)$ & $\mathrm{Li}-\mathrm{Li}(\mathrm{g})$ & $2.977(5)$ \\
$\mathrm{O}(2)-\mathrm{Li}(\mathrm{b})$ & $1.888(4)$ & $\mathrm{Li}-\mathrm{Li}(\mathrm{h})$ & $2.977(5)$ \\
$\mathrm{O}(2)-\mathrm{Li}(\mathrm{g})$ & $1.888(4)$ & $\mathrm{Li}-\mathrm{Li}(\mathrm{i})$ & $3.142(1)$ \\
$\mathrm{Li}-\mathrm{O}(1 \mathrm{a})$ & $1.943(4)$ & $\mathrm{Li}-\mathrm{Li}(\mathrm{i})$ & $3.142(1)$ \\
\hline
\end{tabular}

表 5 原子间键角(度)

\begin{tabular}{lc|lr|lr}
\hline $\mathrm{O}(1)-\mathrm{C}-\mathrm{O}(\mathrm{i})$ & $120.2(1)$ & $\mathrm{Li}(\mathrm{f})-\mathrm{Li}-\mathrm{Li}(\mathrm{i})$ & $96.3(2)$ & $\mathrm{O}(1 \mathrm{a})-\mathrm{Li}-\mathrm{Li}(\mathrm{i})$ & $100.8(2)$ \\
$\mathrm{O}(1)-\mathrm{C}-\mathrm{O}(\mathrm{Ic})$ & $119.6(2)$ & $\mathrm{Li}(\mathrm{f})-\mathrm{Li}-\mathrm{Li}(\mathrm{h})$ & $109.1(1)$ & $\mathrm{O}(1 \mathrm{~b})-\mathrm{Li}-\mathrm{Li}(\mathrm{d})$ & $45.7(1)$ \\
$\mathrm{O}(2)-\mathrm{C}-\mathrm{O}(1 \mathrm{c})$ & $120.2(1)$ & $\mathrm{Ii}(\mathrm{g})-\mathrm{Li}-\mathrm{Li}(\mathrm{h})$ & $113.4(3)$ & $\mathrm{O}(1 \mathrm{~b})-\mathrm{Li}-\mathrm{Li}(\mathrm{f})$ & $119.8(3)$ \\
$\mathrm{C}-\mathrm{O}(1)-\mathrm{Li}(\mathrm{a})$ & $131.4(2)$ & $\mathrm{Li}(\mathrm{g})-\mathrm{Li}-\mathrm{Li}(\mathrm{i})$ & $127.5(2)$ & $\mathrm{O}(1 \mathrm{~b})-\mathrm{Li}-\mathrm{Li}(\mathrm{g})$ & $101.5(2)$ \\
$\mathrm{C}-\mathrm{O}(1)-\mathrm{Li}(\mathrm{c})$ & $114.7(2)$ & $\mathrm{Li}(\mathrm{g})-\mathrm{Li}-\mathrm{Li}(\mathrm{i})$ & $70.9(2)$ & $\mathrm{O}(1 \mathrm{~b})-\mathrm{Li}-\mathrm{Li}(\mathrm{h})$ & $103.9(2)$ \\
$\mathrm{C}-\mathrm{O}(1)-\mathrm{Li}(\mathrm{c})$ & $116.3(1)$ & $\mathrm{Li}(\mathrm{h})-\mathrm{Li}-\mathrm{Li}(\mathrm{i})$ & $109.1(2)$ & $\mathrm{O}(1 \mathrm{~b})-\mathrm{Li}-\mathrm{Li}(\mathrm{i})$ & $37.7(1)$ \\
$\mathrm{C}-\mathrm{O}(2)-\mathrm{Li}(\mathrm{b})$ & $127.5(1)$ & $\mathrm{Li}(\mathrm{h})-\mathrm{Li}-\mathrm{Li}(\mathrm{i})$ & $52.5(2)$ & $\mathrm{O}(1 \mathrm{~b})-\mathrm{Li}-\mathrm{Li}(\mathrm{i})$ & $143.1(1)$ \\
$\mathrm{C}-\mathrm{O}(2)-\mathrm{Li}(\mathrm{g})$ & $127.5(1)$ & $\mathrm{Li}(\mathrm{i})-\mathrm{Li}-\mathrm{Li}(\mathrm{i})$ & $160.5(3)$ & $\mathrm{O}(1 \mathrm{c})-\mathrm{Li}-\mathrm{Li}(\mathrm{d})$ & $119.2(2)$ \\
$\mathrm{Li}(\mathrm{a})-\mathrm{O}(1)-\mathrm{Li}(\mathrm{c})$ & $85.9(2)$ & $\mathrm{O}(1 \mathrm{a})-\mathrm{Li}-\mathrm{O}(1 \mathrm{~b})$ & $94.1(2)$ & $\mathrm{O}(1 \mathrm{c})-\mathrm{Li}-\mathrm{Li}(\mathrm{f})$ & $133.3(2)$ \\
$\mathrm{Li}(\mathrm{a})-\mathrm{O}(1)-\mathrm{Li}(\mathrm{e})$ & $98.9(1)$ & $\mathrm{O}(1 \mathrm{a})-\mathrm{Li}-\mathrm{O}(1 \mathrm{c})$ & $115.1(2)$ & $\mathrm{O}(1 \mathrm{c})-\mathrm{Li}-\mathrm{Li}(\mathrm{g})$ & $74.2(2)$ \\
$\mathrm{Li}(\mathrm{b})-\mathrm{O}(2)-\mathrm{Li}(\mathrm{g})$ & $104.9(2)$ & $\mathrm{O}(1 \mathrm{a})-\mathrm{Li}-\mathrm{O}(2 \mathrm{a})$ & $116.3(2)$ & $\mathrm{O}(1 \mathrm{c})-\mathrm{Li}-\mathrm{Li}(\mathrm{h})$ & $40.1(2)$ \\
$\mathrm{Li}(\mathrm{c})-\mathrm{O}(1)-\mathrm{Li}(\mathrm{e})$ & $103.3(2)$ & $\mathrm{O}(1 \mathrm{~b})-\mathrm{Li}-\mathrm{O}(2 \mathrm{a})$ & $114.2(2)$ & $\mathrm{O}(1 \mathrm{c})-\mathrm{Li}-\mathrm{Li}(\mathrm{i})$ & $132.6(2)$ \\
$\mathrm{Li}(\mathrm{d})-\mathrm{Li}-\mathrm{Li}(\mathrm{f})$ & $103.5(2)$ & $\mathrm{O}(1 \mathrm{~b})-\mathrm{Li}-\mathrm{O}(1 \mathrm{c})$ & $104.2(2)$ & $\mathrm{O}(1 \mathrm{c})-\mathrm{Li}-\mathrm{Li}(\mathrm{i})$ & $39.0(1)$ \\
$\mathrm{Li}(\mathrm{d})-\mathrm{Li}-\mathrm{Li}(\mathrm{g})$ & $66.9(1)$ & $\mathrm{O}(1 \mathrm{c})-\mathrm{Li}-\mathrm{O}(2 \mathrm{a})$ & $111.3(2)$ & $\mathrm{O}(2 \mathrm{a})-\mathrm{Li}-\mathrm{Li}(\mathrm{d})$ & $128.7(3)$ \\
$\mathrm{Li}(\mathrm{d})-\mathrm{Li}-\mathrm{Li}(\mathrm{i})$ & $60.6(2)$ & $\mathrm{O}(1 \mathrm{a})-\mathrm{Li}-\mathrm{Li}(\mathrm{d})$ & $48.4(1)$ & $\mathrm{O}(2 \mathrm{a})-\mathrm{Li}-\mathrm{Li}(\mathrm{f})$ & $37.5(1)$ \\
$\mathrm{Li}(\mathrm{d})-\mathrm{Li}-\mathrm{Li}(\mathrm{i})$ & $137.3(3)$ & $\mathrm{O}(1 \mathrm{a})-\mathrm{Li}-\mathrm{Li}(\mathrm{f})$ & $78.8(1)$ & $\mathrm{O}(2 \mathrm{a})-\mathrm{Li}-\mathrm{L}(\mathrm{g})$ & $140.3(2)$ \\
$\mathrm{Li}(\mathrm{d})-\mathrm{Li}-\mathrm{Li}(\mathrm{h})$ & $144.5(3)$ & $\mathrm{O}(1 \mathrm{a})-\mathrm{Li}-\mathrm{Li}(\mathrm{g})$ & $40.9(1)$ & $\mathrm{O}(2 \mathrm{a})-\mathrm{Li}-\mathrm{Li}(\mathrm{h})$ & $75.6(1)$ \\
$\mathrm{Li}(\mathrm{f})-\mathrm{Li}-\mathrm{Li}(\mathrm{g})$ & $109.1(1)$ & $\mathrm{O}(1 \mathrm{a})-\mathrm{Li}-\mathrm{Li}(\mathrm{h})$ & $152.1(3)$ & $\mathrm{O}(2 \mathrm{a})-\mathrm{Li}-\mathrm{Li}(\mathrm{i})$ & $79.0(2)$ \\
$\mathrm{Li}(\mathrm{f})-\mathrm{Li}-\mathrm{Li}(\mathrm{i})$ & $83.7(2)$ & $\mathrm{O}(1 \mathrm{a})-\mathrm{Li}-\mathrm{Li}(\mathrm{i})$ & $98.2(2)$ & $\mathrm{O}(2 \mathrm{a})-\mathrm{Li}-\mathrm{Li}(\mathrm{i})$ & $89.1(2)$ \\
& & & & & \\
\hline
\end{tabular}

\section{三、结构描述与讨论}

晶体结构由图 1 所示, 碳(C)原子与氧(O)原子组成 $\mathrm{CO}_{3}$ 三角平面分子团,三角平面互椙 平行且近似垂直于 $[001]$ 方向. 与[100]方向成 $6^{\circ}$ 左右的夹角. 锂 $(\mathrm{Li})$ 原子为四配位, $(\mathrm{Li})$ 与 (O)构成 $\mathrm{LiO}$ ，变形的四面体. 每 2 个 $\mathrm{LiO}_{4}$ 四面体以一条共用边相连接. $\mathrm{CO}_{3}$ 三角平面 与 $\mathrm{LiO}_{4}$ 四面体之间以共用角顶氧 (O) 相联结. 氧 $\left(\mathrm{O}_{1}\right)$ 与 1 个碳 $(\mathrm{C})$ 原子和 2 个锂 $\left(\mathrm{Li}^{2}\right)$ 原子相连; 氧 $\left(\mathrm{O}_{2}\right)$ 与 1 个碳 $(\mathrm{C})$ 和 3 个锂 $(\mathrm{Li})$ 相连. 由此而组成一个较为稳定的晶体结 构.

在选择格子类型进行晶胞参数计算时,发现 $H$ 指数存在 $6 a$ 的倍数关系,且在 $[100]$ 方向上 


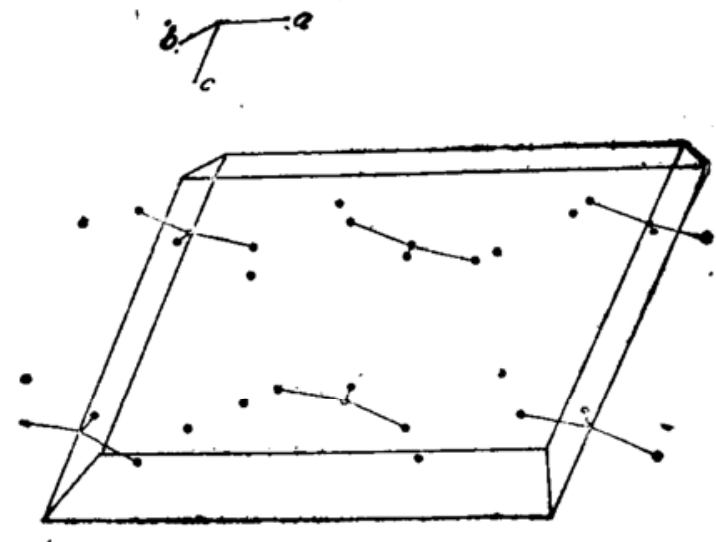

图 1 扎布耶石的晶体结构

亦存在 6 个较弱的衍射点. 因此推测在扎布耶石晶体结构中存在 $6 a$ 超结构的可能性. 有关 超结构工作正在进行中.

致谢：本文实验工作得到中国科学院化学研究所䇥迺钎和郭芳二同志的大力帮助,谨致谢意.

\section{参考 文献}

[1] 郑绾平、刘文高, 矿物学报, 1987,3 .

(2] Zemann, V. J., Acta Cryst., 1957, 10: 664-666.

[3] Effenbeger, H. Und Zemann, V. J.,Zeitschritz für krissallog, aphie, 150 (1979), 133-138.

[4] JCPDS, 1978, 22-1141. 